\title{
BMJ Open Effectiveness of traditional lectures and case methods in Swedish general practitioners' continuing medical education about COPD: a cluster randomised controlled trial
}

\author{
Hanna Sandelowsky, ${ }^{1,2}$ Ingvar Krakau, ${ }^{1,3}$ Sonja Modin, ${ }^{1}$ Björn Ställberg, ${ }^{4}$ \\ Sven-Erik Johansson, ${ }^{1,2}$ Anna Nager ${ }^{1}$
}

To cite: Sandelowsky $\mathrm{H}$, Krakau I, Modin S, et al. Effectiveness of traditional lectures and case methods in Swedish general practitioners' continuing medical education about COPD: a cluster randomised controlled trial. BMJ Open 2018;8:e21982. doi:10.1136/ bmjopen-2018-021982

- Prepublication history and additional material for this paper are available online. To view these files, please visit the journal online (http://dx.doi org/10.1136/bmjopen-2018021982).

Received 31 January 2018 Revised 27 April 2018 Accepted 6 June 2018
Check for updates

(C) Author(s) (or their employer(s)) 2018. Re-use permitted under CC BY-NC. No commercial re-use. See rights and permissions. Published by BMJ.

For numbered affiliations see end of article.

Correspondence to Dr Hanna Sandelowsky; hanna.sandelowsky@sll.se

\section{ABSTRACT}

Objectives To study the effects of continuing medical education (CME) about chronic obstructive pulmonary disease (COPD) for general practitioners (GPs) by comparing two commonly used CME methods with each other and no CME (reference group).

Design A pragmatic cluster randomised controlled trial with primary healthcare centres (PHCCs) as units of randomisation.

Setting, participants and interventions 24 PHCCs in Stockholm County, Sweden, were randomised into two CME intervention arms: case method learning (CM) $(n=12)$ and traditional lectures $(T L)(n=12)$. A reference group without CME $(n=11)$ was recruited separately. GPS $(n=255)$ participated in the study arm to which their PHCC was allocated: $C M, n=87 ; T L, n=93$; and reference, $n=75$. Two 2-hour CME seminars were given in a period of 3 months. Primary outcome measures Changes in scores between baseline and 12 months on a 13-item questionnaire about evidence-based COPD management (0-2 points/question, maximum total score 26 points).

Results 133 (52\%) GPs completed the questionnaire both at baseline and 12 months. Both CM and TL resulted in small yet significantly higher total scores at 12 months than at baseline (CM, 10.34 vs 11.44 ; TL, 10.21 vs 10.91 ; $p<0.05)$; there were few significant differences between these CME methods. At both baseline and 12 months, all three groups' scores were generally high on questions about smoking cessation support and low on those that measured spirometry interpretation skills, interprofessional care and management of multimorbidity.

Conclusions Neither short CM nor short TL CME sessions substantially improve GPs' skills in managing COPD. It is justified to challenge the use of these common CME methods as a strategy for improving GPs' level of knowledge about management of COPD and other complex chronic diseases characterised by multimorbidity. Trial registration number NCT02213809; Results.

\section{INTRODUCTION}

Chronic obstructive pulmonary disease (COPD) is often comorbid with other
Strengths and limitations of this study

- The educational interventions $(2 \times 2$-hour traditional lectures and case-based seminars) studied in this cluster randomised controlled trial are frequently used in real-life Swedish primary care which strengthens the relevance of this study.

- The cluster design of study was a strength, since it decreased potential bias from contamination across individuals at each primary healthcare centre.

- The follow-up investigation 12 months after the intervention was a strength, as it permitted us to observe the effects of the educational interventions beyond the immediate poststudy period.

- The main limitation of the study was the large percentage of non-responders at the end which significantly impaired the ability to draw conclusions.

- Using a written test of knowledge (the general practitioner (GP) questionnaire) to assess the effects of the educational interventions was not optimal because it did not assess change in GPs' behaviours and because the scaling was narrow, decreasing the chances of clear distribution of the scores, which in turn led to few statistically significant changes in the scores.

conditions and is generally underdiagnosed and insufficiently managed in primary care. ${ }^{1}$ Despite improvements in recent years, primary care personnel can still contribute to delays in COPD diagnosis and care through insufficient actions to prevent, detect and manage the disease. ${ }^{1-4}$

In Sweden, the majority of patients with COPD are managed in primary care by general practitioners (GPs) who typically work together with other GPs in group practices and often in co-operation with specialised asthma/COPD nurses and pulmonary rehabilitation personnel. ${ }^{45}$ As GPs are usually the patient's first professional healthcare contact, 
their knowledge about and skills in COPD management need to be up-to-date. ${ }^{6}$ However, there is a considerable gap between current COPD guidelines and what is actually done at GPs' practices. To help transfer theory into practice, more studies on the implementation of COPD guidelines are needed. ${ }^{7}$

Continuing medical education (CME) is a necessary step in implementing optimal care. Although modern research stresses the effectiveness of multiple educational methods in CME, ${ }^{8-10}$ Swedish GPs still often sign up for 1-2-hour lectures, possibly because of their busy schedules. Traditional lectures (TL) are carried out mainly in didactic style with a CME leader as an academic expert. CME that uses case method learning (CM) can be carried out in similar settings and in a similar amounts of time as TL, but the CME leader uses an interactive teaching approach. The professional's perspective on the case described is a central feature in the discussions. ${ }^{11}$ When used in CME in primary care settings, CM has a positive impact on learning. ${ }^{12}{ }^{13}$ In a typical CM seminar, a CME leader facilitates the discussion of one to two patient cases. CM stimulates creative thinking, communication, tolerance for different views, the ability to defend one's own point of view with logic, analysis and decision-making. ${ }^{14}$ It is a learning method that requires previous knowledge and clinical experience in the subject and maturity in the participants.

The current study is part of the PRIMAIR study, a cluster-randomised controlled trial (CRCT) at primary healthcare centres (PHCC) in Stockholm County in 2014-2017. The overall objective of PRIMAIR pertained to the effects of CME on professional COPD practice of individual GPs (GP-related outcomes) and the effects of CME on individual patients (patient-related outcomes). This paper presents only the GP-related outcomes. A detailed description of the GPs' baseline results has been published previously. ${ }^{15}$

The aim of the current study was to compare the effects of CME on the topic of COPD, delivered in the form of praxis-typical, short (1-2-hour) sessions of either CM or TL, tailored for and targeted to GPs. The hypothesis was that CME based on CM leads to greater improvements in GPs' level of knowledge about and skills in COPD management than TL or no CME.

\section{METHODS}

This paper was written in line with the 2010 Consolidated Standards of Reporting Trials (CONSORT) statement: extension to cluster randomised trials. ${ }^{16}$ The CONSORT checklist (online supplementary file 1) and flow chart (figure 1) were used.

Using a computer randomisation program, the authors randomised 24 PHCCs (clusters) in Stockholm, Sweden, into two intervention arms: a CM arm and a TL arm. A reference group of 11 PHCCs (no CME) was recruited separately and was not randomised, as the PHCCs in this group would not receive CME. The GPs participated in the study arm to which their PHCC was allocated.

The pharmaceutical industry did not participate in any part of the study, and we did not offer financial incentives to the participants. As there are no formal requirements for CME for GPs in Sweden, educational credits were not offered.

The CME sessions took place at the PHCCs. Five CME leaders, all GPs competent and experienced in COPD management, ran two 2-hour sessions at each PHCC. The two sessions took place a maximum of 3 months apart. Each PHCC was assigned the same CME leader and CME method (either CM or TL). Thus, four TL leaders taught at two to four PHCCs each, and one CM leader taught at all 12 PHCCs that received CM. John Biggs' educational theory of constructive alignment ${ }^{17}$ was used to align the intended learning outcomes, learning activities and assessments. The intended learning outcomes of the CME were derived from the pre-2015 COPD guidelines ${ }^{2} 18$ and from a 2013 qualitative study of GPs in Stockholm that described barriers to and facilitators of the COPD guideline implementation process. ${ }^{20}$ Each leader adhered to the intended learning outcomes, but the learning activities differed in the CM and LT intervention groups. The leaders were also allowed to use their own presentation materials, such as slide shows and handouts. Apart from a short didactic introduction, participant activating methods (discussions) were the main method used in the CM sessions, whereas the TL sessions followed a traditional didactic style.

The outcome measures for the GPs pertained to individual participants. A GP questionnaire, constructed by the authors and improved after a 'think-aloud' discussion with a group of non-participating GPs, was used to assess GPs' level of knowledge. The paper format questionnaire consisted of five short patient case vignettes and two to three questions per vignette (13 in total). The questions were about 'knowledge/skills' and 'practical management' and consisted of a mixture of multiple choice and open questions. The participants could score 0,1 or 2 points per question. Responses were scored with a premade scoring template. GPs completed the questionnaires immediately prior to and 12 months after the CME sessions, taking 20-30 min each time. At baseline, the GPs replied to the questionnaire on their own without consulting each other. The GPs in the intervention arms did so at the first CME session, and the GPs in the reference group did so at a staff meeting. At 12 months, most GPs, regardless of the study arm, filled in the questionnaire at an ordinary staff meeting. All did so individually. The few GPs who were not present at the staff meeting were contacted by telephone or email and reminded twice. They were allowed to complete the questionnaire on their own. The completed GP questionnaires did not include any information that could identify the GP, so the assessors were blind to cluster allocation. 


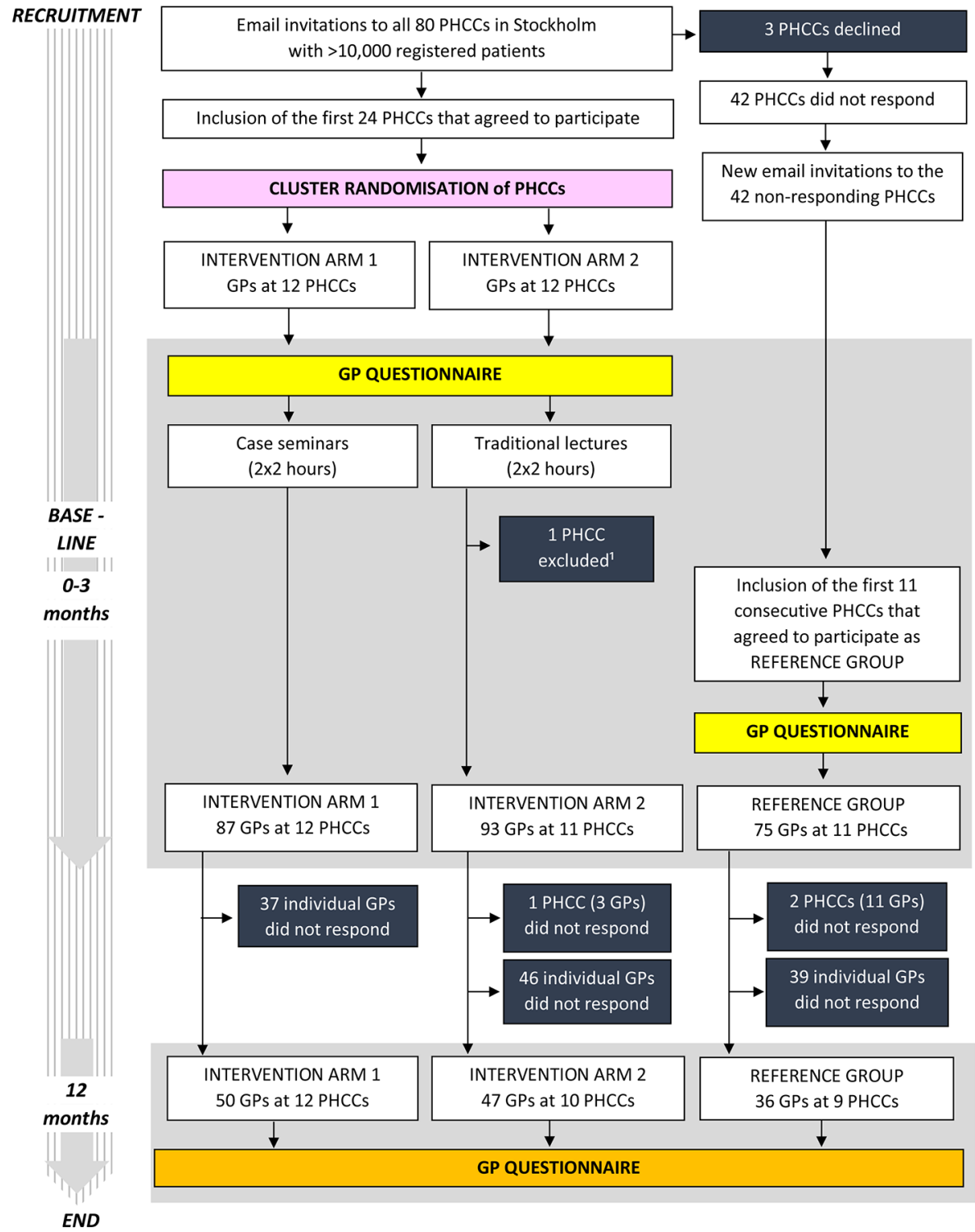

GP, general practitioner; PHCC, primary health care center.

${ }^{1} T$ The main author started working as a GP at this particular PHCC.

Figure 1 Study enrolment, general practitioner part of the PRIMAIR study.

The GP questionnaire with a summary of the intended learning outcomes and the scoring template is found in online supplementary file 2 .

Information about GPs' gender, age, years in the profession and degree (specialist in family medicine or in training to become one) was gathered at baseline. Other information gathered at baseline included data on the PHCC where they worked, such as ownership (county council or private), whether there was a nurse-led asthma/COPD clinic at the PHCC and sociodemographic characteristics of the PHCC's catchment area (Care Need Index; CNI) ${ }^{21}$ The CNI is a deprivation index based on sociodemographic factors, including percentage of older adults living alone, children under age 5, unemployed people, people with low educational status, single parents, high mobility and foreign-born people. A high CNI score indicates high sociodemographic burden. The mean CNI score of PHCC catchment areas in Stockholm County is 2.49.

GP sample size was determined by the power calculation of the patient sample size in the PRIMAIR Study which was determined to be 230 patients with COPD in Global Initiative for Chronic Obstructive Lung Disease (GOLD) stages $2-3^{2}$ in each arm. To keep the number of clusters reasonable, we chose to invite only PHCCs with more than 10000 registered patients $(n=80)$. We estimated that 10-12 PHCCs were needed per arm to achieve sufficient statistical power for the patient sample. Accordingly, the number of GPs was determined by the number of PHCCs we included. Unequal cluster sizes (5-10 GPs) were expected because of variations in staff numbers at baseline and dropouts at 12 months. The intraclass correlation coefficient (ICC) was set at 
0.01 based on earlier studies on cluster randomisations in primary care. ${ }^{22-24}$

\section{Statistics}

We performed the statistical analysis with STATA V.14 (Stata 2015; Stata Statistical Software: Release 14) and SPSS (IBM, Release 2013. IBM SPSS Statistics for Windows, V.23.0). We computed summary statistics such as means, proportions and measures with standard parametric methods. We used a McNemar test to compare matched pairs of scores per question at baseline and 12 months for proportions of GPs who scored ' 0 points' vs ' 1 or 2 points'. We used clttest and xtreg (adjusting for cluster) to analyse differences in total scores within the study arms and for adjusting for 'total scores at baseline', 'CNI' and 'years in profession'. A transition model, adjusted for clusters, was applied to analyse associations between items (' 0 points' or ' 1 or 2 points') and study arms at baseline and 12 months which also provided ORs and their $95 \%$ CIs. In a transition model, the outcome variable at a previous time point is included as a fixed effect covariate. We condition the response at time $\mathrm{j}$ on the response at time $\mathrm{j}-1$. ICC was estimated by xtlogit. $\mathrm{P}$ values $<0.05$ were considered indicative of statistical significance.

A detailed description of the methodology and interventions is found in the study protocol. ${ }^{25}$

The study was registered at www.clinicaltrials.gov on 10 August 2014, Identifier NCT02213809. The first participant was enrolled on 14 August 2014.

\section{Patient and public involvement}

No patients or public were involved in the study.

\section{RESULTS}

\section{Description of the participants}

At baseline, 207 GPs attended the CME sessions. Twenty-seven of them did not agree to participate in the study and thus did not fill in the GP questionnaire. Eightyseven of 100 GPs $(87 \%)$ in the CM arm and 93 of 107 GPs $(87 \%)$ in the TL arm agreed to participate in the study. The reference group consisted of 75 GPs. The majority of the GPs who did not agree to participate worked at a PHCC without a nurse-led asthma/COPD clinic. They did not differ from the participants regarding age, gender, years in profession, educational degree or PHCC's CNI scores or ownership form.

Of the 255 participants who responded to the questionnaire at baseline, $122(48 \%)$ did not respond again at 12 months ('non-responders'). The remaining 133 GPs were the final participants ('responders'). There were no significant differences between any of the groups studied (the two intervention arms and the reference group) in the proportions of responders and non-responders. A higher percentage of the non-responders than responders were employed at PHCCs in socially deprived areas of Stockholm $(p<0.05)$. The characteristics of the responders and non-responders are seen in table 1.
A higher percentage of GPs in the CM arm than the TL arm and the reference group worked at a PHCC with a nurse-led asthma/COPD clinic (64\% vs $36 \%-38 \%, p=0.012$ ). A higher percentage of GPs in the reference group than the CM and TL arms worked at privately run PHCCs $(72 \%$ vs $32 \%-42 \%, \mathrm{p}=0.001)$. The means for gender, age, years in profession and CNI scores did not differ significantly between the GPs in the groups studied (the two intervention arms and the reference group), and the participants were generally representative for Swedish GPs with regard to these characteristics. ${ }^{26}$

\section{Scores}

Total scores: within and between the arms

After adjustment for the clusters (ie, PHCCs) and mean scores at baseline, the mean scores in both intervention arms were significantly higher at 12 months than at baseline (CM: 10.34 vs 11.44; TL: 10.21 vs $10.91 ; \mathrm{p}<0.05$ ) (figure 2). There was no statistically significant difference between the improvement in the CM and TL arms. No significant changes in scoring over time were observed in the reference group. All the non-responders had significantly lower mean baseline scores than the responders (9.11 vs 10.47 , $\mathrm{p}=0.003$ ). At baseline, the GPs who worked at PHCCs in the most socially deprived areas (CNI 2.29-5.05, 21\% of all GPs) had lower mean scores than the others $(8.50$ vs $10.32, \mathrm{p}=0.000)$, and the non-responders in the deprived areas scored lowest of all non-responders ( 7.98 vs $9.71, p=0.007)$. Scores were unrelated to whether or not there was a nurse-led asthma/ COPD clinic at the PHCC.

\section{Scores on individual questions: comparison of the arms}

Table 2 compares the results for each of the 13 questions in the two intervention arms and the reference group by providing ORs of 'scoring 1 or 2 points at 12 months'. The TL arm had a statistically significantly higher OR of 'scoring 1 or 2 points at 12 months' than the reference group on two of the questions: the question about the follow-up of stable patients (question 9 ) and the question about multimorbidity in a patient with airway symptoms (question 13). On the question about smoking cessation support for patients who were motivated to quit smoking (question 6), the TL arm had a higher OR of 'scoring 1 or 2 points at 12 months' than the CM arm. The CM arm's ORs were not significantly higher for any of the questions than the TL arm or reference group's ORs. Regarding the effects of intracluster conditions, we found three significant ICCs (questions 1, 10 and 12), all of them were approximately 0.10 (CIs could not be estimated because no SE was available).

Scores on individual questions: within the arms

For 10 of 13 questions, there was no significant difference between baseline and 12 months in the proportion of participants who scored 1 or 2 points and who scored 0 points (figure 3). Scores on two questions 
Table 1 The main characteristics of the participants in the two intervention arms and reference group

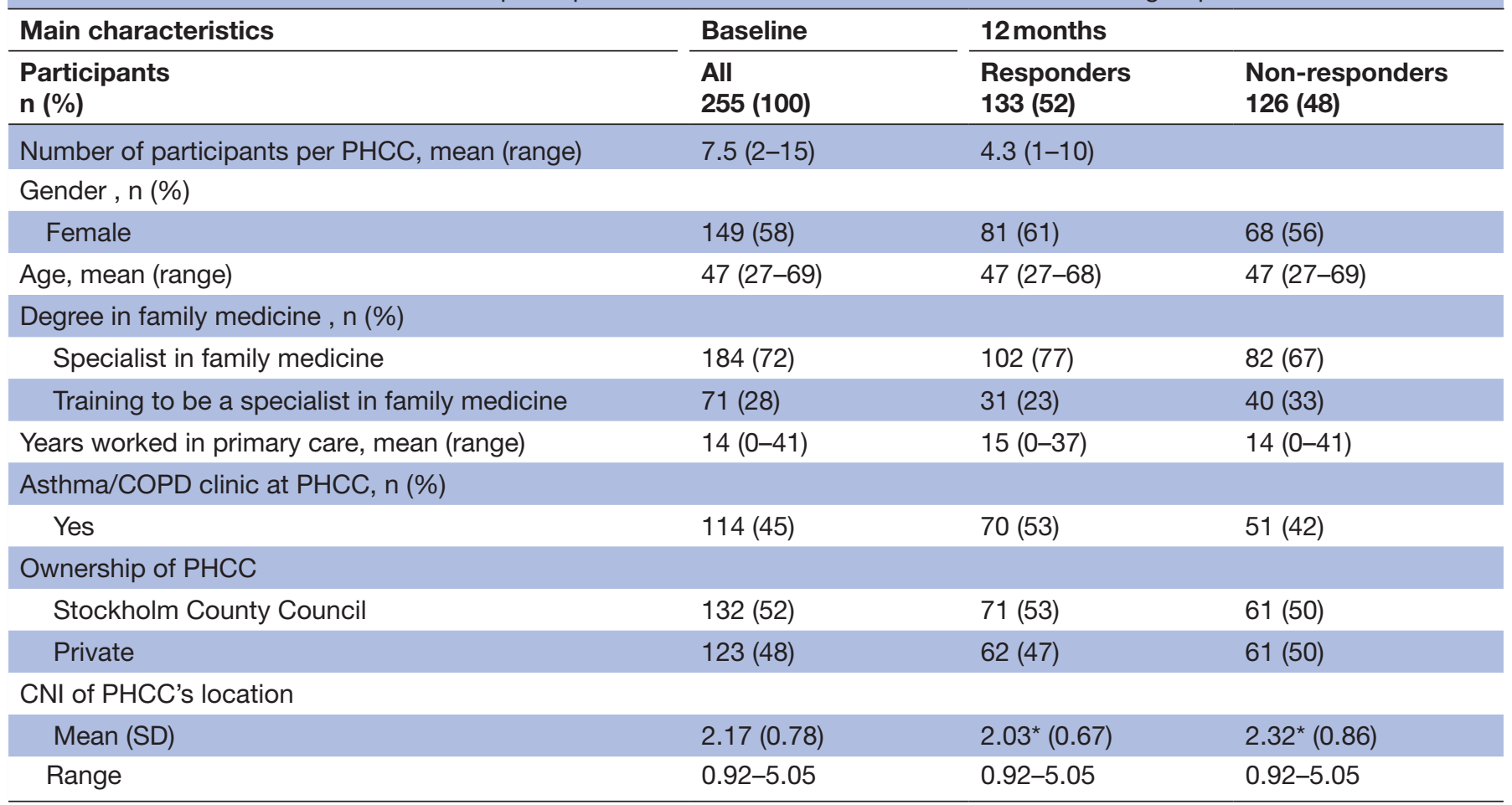

The CNI is based on sociodemographic factors, including percentage of older adults living alone, children under age 5, unemployed people, people with low educational status, single parents, high mobility and foreign-born people. High $\mathrm{CNI}=$ high sociodemographic burden; mean $\mathrm{CNI}$ in Stockholm County=2.49.

${ }^{*} \mathrm{P}$ of the difference between responders and non-responders $<0.05$.

$\mathrm{CNI}$, Care Need Index; COPD, chronic obstructive pulmonary disease; PHCC, primary healthcare centre.

improved significantly (CM arm: question 2, spirometry interpretation; TL arm: question 9, follow-up of stable patients), and scores on one question dropped significantly (reference group: question 13, multimorbidity in a patient with airway symptoms) (figure $4 \mathrm{~A}-\mathrm{C}$ ).

\section{DISCUSSION}

\section{Main findings}

The results of our study do not bear out the hypothesis that CM leads to greater improvements in GPs' level of COPD-related knowledge and skills than TL. However, the hypothesis that CM would be superior to no intervention

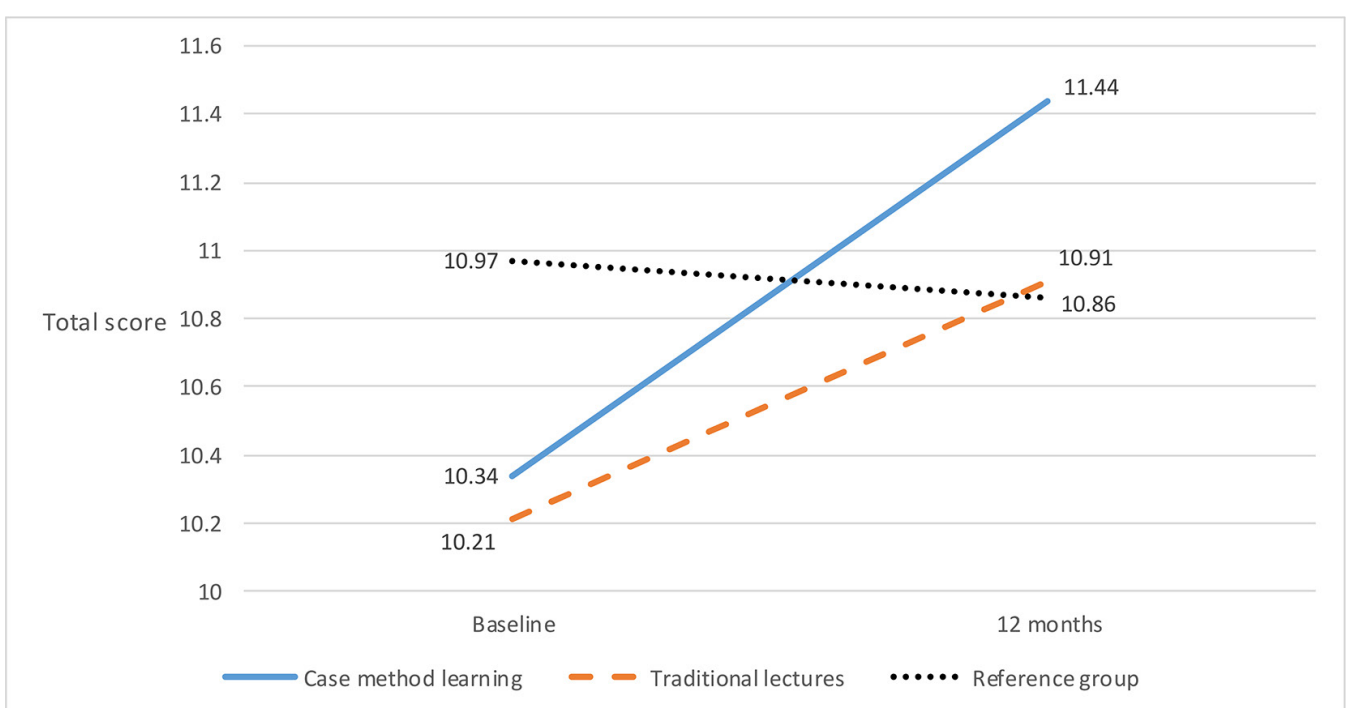

Figure 2 Development of total scores in the two intervention arms and reference group over time. Total score minimum $=0$ points, maximum $=26$ points. 
Table 2 Comparison of the ORs and $95 \%$ Cls of scoring 1 or 2 rather than 0 points in the two intervention arms (case methods, CM; traditional lectures, TL) and the reference group (no continuing medical education) 12 months after the intervention

\begin{tabular}{ll}
\hline OR & \\
\hline $\mathrm{CM}$ and TL vs reference group & $\begin{array}{l}\mathrm{CM} \text { vs TL } \\
(95 \% \mathrm{Cl})\end{array}$ \\
\cline { 2 - 2 } & $(95 \% \mathrm{Cl})$ \\
\hline
\end{tabular}

\begin{tabular}{|c|c|c|c|c|c|}
\hline Question & CM & TL & $\begin{array}{l}\text { Reference } \\
\text { group }\end{array}$ & CM & TL \\
\hline 1. Diagnostic procedures & 0.55 (0.22 to 1.40$)$ & 0.55 (0.21 to 1.42$)$ & 1 & 1.00 (0.43 to 2.31$)$ & 1 \\
\hline 2. Spirometry interpretation & 1.29 (0.53 to 3.10$)$ & 0.61 (0.24 to 1.55$)$ & 1 & 2.10 (0.90 to 4.95$)$ & 1 \\
\hline $\begin{array}{l}\text { 3. Smoking cessation (unmotivated } \\
\text { patients) }\end{array}$ & $N A^{*}$ & & & & \\
\hline 4. Acute exacerbation (treatment) & $1.40(0.57$ to 3.45$)$ & 0.77 (0.31 to 1.96$)$ & 1 & 1.81 (0.77 to 4.24$)$ & 1 \\
\hline 5. Acute exacerbation (follow-up) & NAt & & & & \\
\hline 6. Smoking cessation (motivated patients) & $0.41(0.14$ to 1.24$)$ & 1.35 (0.39 to 4.69$)$ & 1 & 0.30 (0.10 to 0.88$)$ & 1 \\
\hline 7. Maintenance treatment & $1.04(0.42$ to 2.54$)$ & $0.72(0.30$ to 1.74$)$ & 1 & 1.44 (0.63 to 3.29$)$ & 1 \\
\hline 8. Comorbidity: heart failure & 2.46 (0.79 to 7.66$)$ & 1.70 (0.61 to 4.95$)$ & 1 & 1.45 (0.57 to 3.67$)$ & 1 \\
\hline 9. Follow-up (stable patients) & 2.37 (0.96 to 5.86$)$ & 4.48 (1.51 to 13.3$)$ & 1 & 0.53 (0.20 to 1.40$)$ & 1 \\
\hline 10. Interprofessional interventions & 1.82 (0.59 to 5.61$)$ & 1.42 (0.45 to 4.49$)$ & 1 & 1.29 (0.50 to 3.31$)$ & 1 \\
\hline 11. Suspected respiratory failure & 1.51 (0.62 to 3.72$)$ & 0.97 (0.39 to 2.41$)$ & 1 & 1.57 (0.68 to 3.62$)$ & 1 \\
\hline 12. Multimorbidity, no airway symptoms & 1.36 (0.54 to 3.40$)$ & 0.97 (0.39 to 2.43 ) & 1 & 1.39 (0.60 to 3.24$)$ & 1 \\
\hline 13. Multimorbidity, airway symptoms & $1.34(0.53$ to 3.37$)$ & 2.64 (1.06 to 6.60$)$ & 1 & 0.51 (0.22 to 1.15$)$ & 1 \\
\hline
\end{tabular}

All measures are adjusted for clusters (primary healthcare centres) and total scores at baseline.

${ }^{*}$ OR not applicable because there was no convergence in the model.

†OR not applicable because the model was questionable.

NA, not applicable.

was confirmed. Both CM and TL led to small, yet significant, improvements in Swedish GPs' levels of knowledge of COPD and COPD management skills. Neither of the CME methods was more effective than the other. GPs' baseline level of knowledge was low, and improvements at 12 months were generally modest. Moreover, GPs' level of knowledge was unrelated to whether or not they worked at a PHCC with a nurse-led asthma/COPD clinic.

\section{Over time, strong areas of knowledge remained strong, and weak areas weak}

For the most part, the differences between strong and weak areas of knowledge about COPD were maintained over time. We were not surprised by the high level of knowledge about smoking cessation support, as most GPs are well aware of and do not question the importance of quitting smoking. However, there is a gap between what GPs know and what they do: earlier research has shown that doctors rarely take active measures to help patients quit smoking. ${ }^{27}$ The preliminary results of a questionnaire to randomly selected patients with COPD at the PHCCs participating in this study indicate that approximately $60 \%$ of the patients who are current smokers or have quit smoking in the last 5 years $(\mathrm{n}=382)$ state they have not been offered smoking cessation support, and $80 \%$ state they have not been given such support by their GPs or nurses (Sandelowsky, unpublished data). As the benefits of smoking cessation far outweigh the benefits of diagnosing new cases or providing pharmacological treatments for COPD, ${ }^{28}$ the GP's role as a motivator and authority in patient education should not be overlooked and needs continuous attention in CME situations.

Insufficient skills in spirometry interpretation may be one of the major causes of problems with implementing evidence-based COPD practice. ${ }^{29} \mathrm{CM}$ involved active participation in training spirometry interpretation and discussing spirometry results which may explain the improvements in responses to the question that measured spirometry interpretation skills. However, this was the only question on spirometry interpretation, and conclusions based on the responses to one question may not be reliable. TL positively affected replies to the questions on smoking cessation support to motivated patients, follow-up of patients with stable COPD and management of airway symptoms in multimorbid patients. We did not investigate whether this finding was due to factors related to the CME leaders (ie, uneven focus on the different intended learning outcomes) or to the didactic lecturing style.

Neither CME method led to significant improvements in managing COPD in patients with multimorbidities and comorbidities, managing acute exacerbation under time pressure, managing patients who lack motivation to quit smoking or handling patients whose focus during 


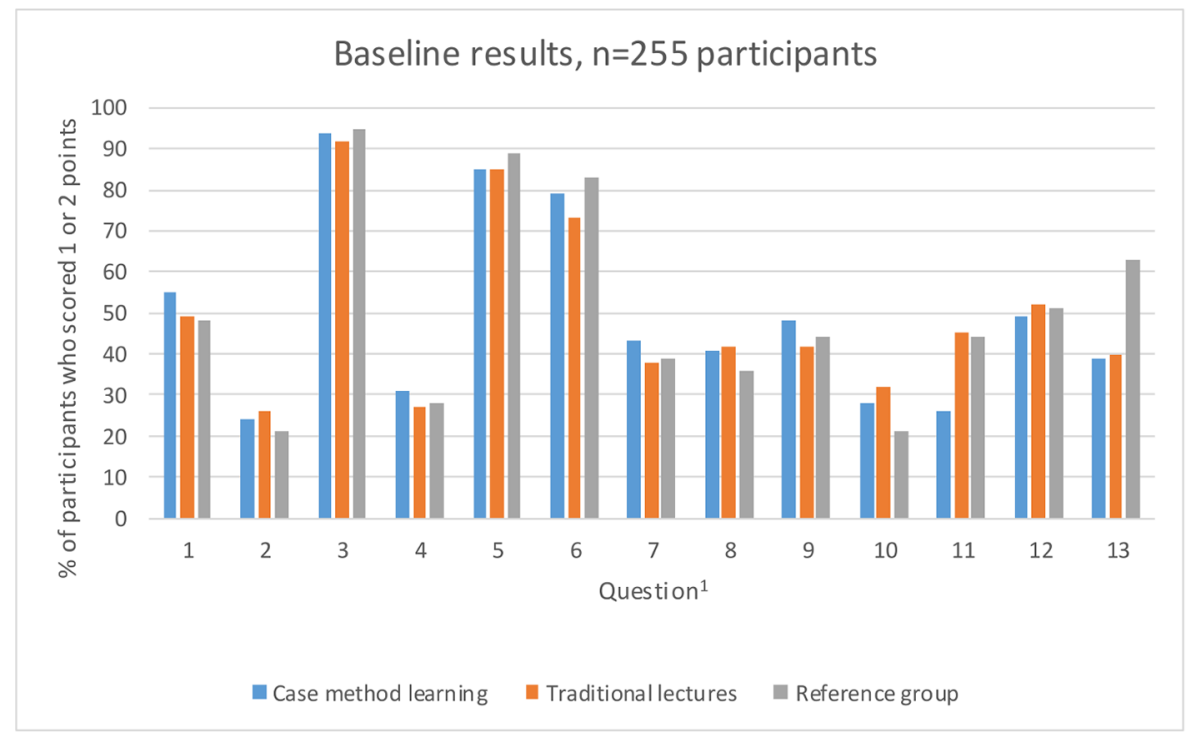

${ }^{1}$ Questions: 1) Diagnostic procedures; 2) Spirometry interpretation; 3) Smoking cessation (unmotivated patients); 4) Treatment of acute exacerbation; 5) Follow-up of acute exacerbation; 6) Smoking cessation (motivated patients); 7) Maintenance treatment of COPD (GOLD B patients); 8) Heart failure medication for patients with COPD; 9) Follow-up of patients with stable COPD; 10) Inter-professional interventions; 11) Managing a suspected respiratory failure; 12) Multi-morbidity in COPD patients without obvious symptoms from airways or COPD comorbidities (an annual checkup); 13) Multi-morbidity in COPD patients with symptoms from airways and/or COPD comorbidities (an annual check-up)

Figure 3 Baseline results per question in the two intervention arms and reference group, presented as per cent of participants who scored 1 or 2 points. Each response was given a score of between 0 and 2 points; the highest possible score was 2 points. On questions 2, 4, 7, 10 and 11, only two scores were possible: 0 or 2 points. COPD, chronic obstructive pulmonary disease.

the consultation is something other than COPD. Thus, these typical real-life conditions and problems in Swedish primary care, which represent important obstacles to implementing guidelines, ${ }^{20}$ remain difficult to overcome with two short sessions of CME, regardless of whether lectures or case methods are used.

\section{Comparison with previous studies}

Kiessling et al found that using CM to implement evidence-based practice in primary care was associated with decreased mortality in patients with coronary heart disease in Sweden 10 years after the training had taken place. $^{12}$ The educational meetings in Kiessling's study were carried out similarly to those in our study; that is, as short CM seminars for GPs at their workplaces, led by an external facilitator. COPD may be a more complex health issue than cardiovascular diseases, and evidence-based management may thus be more complex to implement. The complexity of COPD typically includes disabling comorbidities, ${ }^{1}$ confronting lifestyle choices (mainly smoking), low patient motivation to adhere to treatment, ${ }^{20}{ }^{30}$ GPs' negative views of COPD,${ }^{20}$ consequences of COPD in patients' family lives ${ }^{31}$ and the crucial role of interprofessional care. ${ }^{32}$

We are not alone in finding that CM sessions brief enough for busy GPs to attend are of limited effectiveness. A 2016 Swedish study about the effectiveness of CM in CME for GPs on the topic of childhood asthma used CM similar to those in our study. That intervention had no effects on prescriptions of antiasthmatic drugs for children. ${ }^{33}$

In line with theories of adult learning, the American College of Chest Physicians recommends multiple teaching techniques, such as CM, audience response system, lectures, hands-on demonstrations, discussion groups and role playing to effectively change physician knowledge, performance and clinical outcomes. ${ }^{8}$ In fact, two previous CME studies from the USA, by Drexel et al and Adams et al, found that CME had positive effects on GPs' management of COPD when used as one of multiple educational methods, including a combination of short didactic lectures, case discussions, spirometry workshops and inhaler demonstrations. ${ }^{1334}$ Moreover, Adams et al observed positive outcomes following interactive and collaborative CME for multidisciplinary participants, which is particularly relevant, as the current Swedish guidelines strongly recommend interprofessional COPD care. ${ }^{32}$ However, the follow-up measurements in both the Adams $e t a l$ and Drexel $e t a l$ studies were made shortly after the CME intervention and thus do not provide information about the sustainability of results. Additionally, in the Drexel $e t$ al study, no preintervention measurements were performed which limits the researcher's ability to evaluate the effects of the CME.

\section{Methodological considerations}

Strengths and weaknesses

One strength of this study was the cluster randomisation of the PHCCs, which reduced the likelihood of possible 

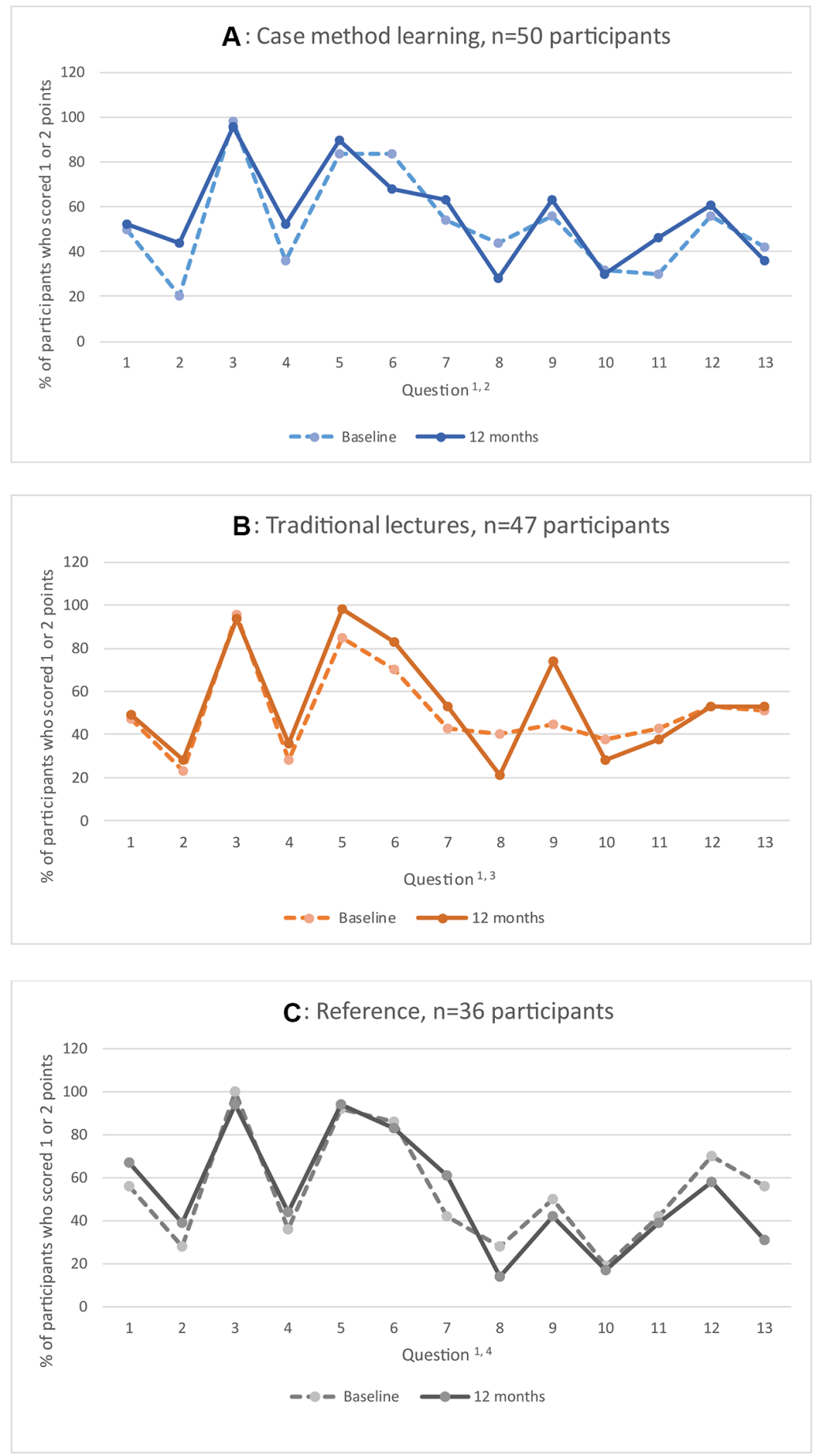

${ }^{1}$ Questions: 1) Diagnostic procedures; 2) Spirometry interpretation; 3) Smoking cessation (unmotivated patients); 4) Treatment of acute exacerbation; 5) Follow-up of acute exacerbation; 6) Smoking cessation (motivated patients); 7) Maintenance treatment of COPD (GOLD B patients); 8) Heart failure medication for patients with COPD; 9) Follow-up of patients with stable COPD; 10) Inter-professional interventions; 11) Managing a suspected respiratory failure; 12) Multi-morbidity in COPD patients without obvious symptoms from airways or COPD comorbidities (an annual check-up); 13) Multimorbidity in COPD patients with symptoms from airways and/or COPD comorbidities (an annual check-up)

${ }^{2}$ In figure $4 a, p<0.05$ for question 2

${ }^{3}$ In figure $4 b, p<0.05$ for question 9

${ }^{4}$ In figure $4 c, p<0.05$ for question 13

Figure 4 (A-C) Changes over time in the scores per question in each group studied, presented as per cent of participants who scored 1 or 2 points. Each response was given a score of between 0 and 2 points; the highest possible score was 2 points. On questions 2, 4, 7, 10 and 11, only two scores were possible: 0 or 2 points. COPD, chronic obstructive pulmonary disease. 
contamination across individuals at each PHCC (cluster). Including a reference group enabled us to compare knowledge gained through our interventions with information gained through other sources during the study period. Using a transition model in the analysis of data was a strength because of its simplicity. The statistically significant ICCs we found for the questions were higher than expected, indicating that intracluster conditions may have affected the GPs' results in some of the individual questions more than assumed prior to the intervention. This information may assist researchers conducting CRCTs in similar environments in the future. Our assessment indicated that there were practically no interactions between the clusters that could have biased the results.

Swedish GPs report they have little time for CME because of heavy workloads due to time constraints, staffing problems and financial incentives at the PHCC that encourage multiple short visits. ${ }^{26}$ A previous study in a similar setting found that primary healthcare professionals appreciate CME outreach visits. ${ }^{35}$ The CME outreach visits in the current study were thus another strength, as they enabled the GPs to attend the CME sessions despite their busy schedules. Another strength was the choice to conduct the follow-up measurement 12 months after the intervention, which enabled us to describe the sustained effects of the interventions.

The recruitment of the reference group deviated from optimal CRCT design. Thus, some caution should be used when interpreting the results for the reference group. Another limitation of our study was the potential for reporting bias at 12 months due to non-response. High non-response impaired the statistical power of the 12-month analysis and decreased our ability to generalise the results. A high dropout percentage was also observed in the Adams et al CME study. The high dropout rates in the two studies may reflect the strenuous working conditions GPs often experience: reminders had practically no effect on response rate. Non-responders may also have been uncomfortable reporting their potential lack of knowledge gain after the CME. Moreover, it was alarming to find that many of the non-responders worked in deprived urban areas where smoking and COPD are common. ${ }^{36} 37$

A source of bias may have been the minor overlapping of the two pedagogical methods. Although we focused on providing didactic lectures in the TL sessions, some natural interplay may have taken place. On the other hand, CM consists of a mixture of didactic and participatory learning methods. The TL sessions were taught by four different CME leaders and the CM sessions were facilitated by one, which may have further biased the results, as the TL leaders may have stressed different content.

To achieve deeper knowledge via CM, participants must have previous knowledge and clinical experience in the subject area. Analysis indicated that GPs' baseline level of knowledge about COPD was surprisingly low. We reason that it may have been too low to enable them to take full advantage of the CM learning opportunity. Thus, a sequence of different CME interventions in which $\mathrm{CM}$ was not the first step might have been more effective. In retrospect, we could have improved the teaching activities by conducting a pilot survey to measure GPs' knowledge prior to designing the intervention or by designing the teaching activities after collecting and analysing the baseline data.

The GP questionnaire had strengths and limitations. A 'think-aloud' discussion with a group of non-participating GPs helped us improve the five case vignettes' understandability and relatability, increasing the chances of valid replies to the questions. However, written descriptions, such as in case vignettes and multiple-choice answers, always involve a risk of misinterpretation, and thus of biased replies. We suspect this could have happened in question 8 , as it was expressed in inverted (negative) wording. Another limitation of the study was that the questionnaire was the only assessment method we used, so we were unable to assess whether the GPs' behaviours changed in practice. The use of mixed methods would have helped ensure the best possible assessment validity. ${ }^{38}$ The narrow, $0-2$ point scale, together with strict scoring requirements, may have contributed to difficulties in differentiating the participants' results. We tried to minimise the testing bias that can occur when the same questionnaire is administered twice by not revealing the answers and by using paper questionnaires to disable digital distribution of the questionnaire. Finally, participants may have received information about COPD through other channels during the study period.

\section{Implications and future research}

The findings of this study can be useful in developing CME interventions that are feasible to implement in a busy primary care practice and that target the management of complex, chronic health issues. ${ }^{26} 39$ The particularly low competence in the subject of COPD among GPs in socially deprived areas sends an important message to policy-makers, as smoking and COPD are particularly prevalent in these areas. ${ }^{36} 37$

An evaluation of patient-related outcomes before and after the CME intervention is under way as a part of the PRIMAIR study. Future research could evaluate the effects of a sequel to our CME intervention that incorporates other educational methods and/or angles the focus towards interprofessional learning activities to support team-based COPD care in primary care. However, such interventions would likely need to be longer than two short sessions, which would make them challenging to implement in primary care. As many people now acquire knowledge via digital media, future research could also investigate the effects of easy-to-access online handbooks and guides as support for GPs in clinical decision-making.

\section{CONCLUSION}

GPs' levels of knowledge of and skills for COPD were low at baseline, and the effects of both case methods and TL were equally modest. Thus, these common educational methods alone may not be sufficient to substantially 
improve GPs' level of knowledge and management of COPD. Critics are justified in challenging the use of a single CME method in short sessions as a strategy for improving management of patients with COPD or other complex chronic diseases characterised by multimorbidity.

\section{Author affiliations}

${ }^{1}$ NVS, Section for Family Medicine and Primary Care, Karolinska Institutet,

Stockholm, Sweden

${ }^{2}$ Academic Primary Health Care Centre, Stockholm, Sweden

${ }^{3}$ Division of Clinical Epidemiology, Department of Medicine, Karolinska Institutet, Stockholm, Sweden

${ }^{4}$ Department of Public Health and Caring Sciences, Family Medicine and Preventive Medicine, Uppsala University, Uppsala, Sweden

Acknowledgements The authors would like to thank Scientific Editor Kimberly Kane for useful comments on the text and Senior Lecturer Ingrid Hylander for advice on the analysis of the questionnaires.

Contributors HS, IK, SM, BS and AN conceived and designed the study. HS and BS were two of the five CME leaders. HS collected the data. HS, BS, S-EJ and AN analysed the data. HS wrote the paper. All authors edited, revised and approved the final manuscript.

Funding This work was supported by employment in and grants from the Stockholm County Council (grand register number LS 1110-1339, LS 1301-0078 and LS 1411-1373), employment in Dalarna County Council, and an unrestricted research grant from AstraZeneca Inc.

Competing interests HS has received honoraria for educational activities from Boehringer Ingelheim, Novartis, AstraZeneca, and TEVA and an unrestricted research grant from AstraZeneca. AN has received compensation for educational activities from AstraZeneca and SM from Novartis. BS has received honoraria for educational activities and lectures from AstraZeneca, Boehringer Ingelheim, GlaxoSmithKline, Meda, Novartis, and TEVA and has served on advisory boards arranged by AstraZeneca, Novartis, Meda, TEVA, GlaxoSmithKline, and Boehringer Ingelheim.

\section{Patient consent Not required}

Ethics approval Regional Ethical Review Board of Stockholm (ref 2013/232-31/5).

Provenance and peer review Not commissioned; externally peer reviewed.

Data sharing statement Data analysed in this study are available from the corresponding author in response to requests that comply with ethical principles of good research.

Open access This is an open access article distributed in accordance with the Creative Commons Attribution Non Commercial (CC BY-NC 4.0) license, which permits others to distribute, remix, adapt, build upon this work non-commercially, and license their derivative works on different terms, provided the original work is properly cited, appropriate credit is given, any changes made indicated, and the use is non-commercial. See: http://creativecommons.org/licenses/by-nc/4.0/.

\section{REFERENCES}

1. Ställberg B, Janson C, Johansson G, et al. Management, morbidity and mortality of COPD during an 11-year period: an observational retrospective epidemiological register study in Sweden (PATHOS). Prim Care Respir J 2014;23:38-45.

2. Global Initiative for Chronic Obstructive Lungdisease. $2013 \mathrm{ttp}: / /$ www.goldcopd.com/ (cited 17 Jan 2018).

3. Lindberg A, Bjerg A, Bjerg-Bäcklund A, et al. Prevalence and underdiagnosis of COPD by disease severity and the attributable fraction of smoking Report from the Obstructive Lung Disease in Northern Sweden Studies. Respir Med 2006;100:264-72.

4. Lisspers $\mathrm{K}$, Johansson $\mathrm{G}$, Jansson $\mathrm{C}$, et al. Improvement in COPD management by access to asthma/COPD clinics in primary care: data from the observational PATHOS study. Respir Med 2014;108:1345-54.

5. Arne M, Emtner M, Lisspers K, et al. Availability of pulmonary rehabilitation in primary care for patients with COPD: a crosssectional study in Sweden. Eur Clin Respir J 2016;3:31601.
6. Lisspers K, Ställberg B, Hasselgren M, et al. Primary health care centres with asthma clinics: effects on patients knowledge and asthma control. Prim Care Respir J 2010;19:37-44.

7. Pinnock $\mathrm{H}$, Thomas M, Tsiligianni I, et al. The International Primary Care Respiratory Group (IPCRG) Research Needs Statement 2010. Prim Care Respir J 2010;19(Suppl 1):S1-20.

8. Davis D, Galbraith R. American College of Chest Physicians Health and Science Policy Committee. Continuing medical education effect on practice performance: effectiveness of continuing medical education: American College of Chest Physicians Evidence-Based Educational Guidelines. Chest 2009;135(3 Suppl):42S-8.

9. Forsetlund L, Bjorndal A, Rashidian A, et al. Continuing education meetings and workshops: effects on professional practice and health care outcomes. Cochrane Database Syst Rev 2009;2:CD003030.

10. Grimshaw JM, Eccles MP, Lavis JN, et al. Knowledge translation of research findings. Implement Sci 2012;7:50.

11. Nordquist J. Att undervisa med case $i$ utbildningar inom hälso- och sjukvården. Karolinska Institutet, Mecical Care Centre: Stockholm: 2009.

12. Kiessling A, Lewitt M, Henriksson P. Case-based training of evidence-based clinical practice in primary care and decreased mortality in patients with coronary heart disease. Ann Fam Med 2011;9:211-8.

13. Drexel C, Jacobson A, Hanania NA, et al. Measuring the impact of a live, case-based, multiformat, interactive continuing medical education program on improving clinician knowledge and competency in evidence-based COPD care. Int J Chron Obstruct Pulmon Dis 2011;6:297-307.

14. Mauffette-Leenders LA, Erskine JA, Leenders MR, et al. London [Ont ]: Richard Ivey School of Business, The University of Western Ontario 1997.

15. Sandelowsky H, Natalishvili N, Krakau I, et al. COPD management by Swedish general practitioners - baseline results of the PRIMAIR study. Scand J Prim Health Care 2018;36:5-13.

16. Campbell MK, Piaggio G, Elbourne DR, et al. CONSORT Group. Consort 2010 statement: extension to cluster randomised trials. BMJ 2012;345:e5661.

17. Biggs John CT, ed. Teaching for Quality Learning at University: The McGraw-Hill Companies, 2011.

18. National Board of Health and Welfare. Socialstyrelsens riktlinjer för vård av astma och kroniskt obstruktiv lungsjukdom (KOL). Faktadokument och beslutsstöd förprioriteringar 2004.

19. Swedish Medical Products Agency. Läkemedelsverkets riktlinjer för behandling av kroniskt obstruktiv lungsjukdom. 2009 https:// lakemedelsverket.se/upload/halso-och-sjukvard/behandlingsrekom mendationer/KOL_rek_webb.pdf (cited 17 Jan 2018).

20. Sandelowsky H, Hylander I, Krakau I, et al. Time pressured deprioritization of COPD in primary care: a qualitative study. Scand $J$ Prim Health Care 2016;34:55-65.

21. Sundquist K, Malmström M, Johansson SE, et al. Care Need Index, a useful tool for the distribution of primary health care resources. $J$ Epidemiol Community Health 2003;57:347-52.

22. Adams G, Gulliford MC, Ukoumunne OC, et al. Patterns of intracluster correlation from primary care research to inform study design and analysis. J Clin Epidemiol 2004;57:785-94.

23. Killip S, Mahfoud Z, Pearce K. What is an intracluster correlation coefficient? Crucial concepts for primary care researchers. Ann Fam Med 2004;2:204-8

24. Hayes RJ MH, Trials CR, Baton B. FL: Chapman \& Hall/CRC. Taylor \& Francis Group 2009.

25. Sandelowsky H, Krakau I, Modin S, et al. Case Method in COPD education for primary care physicians: study protocol for a cluster randomised controlled trial. Trials 2017;18:197.

26. Pettersson S. Swedish Medical Association's Primary Care Survey] In Swedish. Stockholm: Swedish Medical Association, 2015:16-59.

27. Stead M, Angus K, Holme I, et al. Factors influencing European GPs' engagement in smoking cessation: a multi-country literature review. Br J Gen Pract 2009;59:682-90.

28. Black WC, Gareen IF, Soneji SS, et al. Cost-effectiveness of CT screening in the National Lung Screening Trial. N Engl J Med 2014;371:1793-802.

29. Foster JA, Yawn BP, Maziar A, et al. Enhancing COPD management in primary care settings. MedGenMed 2007;9:24.

30. Roddy E, Antoniak M, Britton J, et al. Barriers and motivators to gaining access to smoking cessation services amongst deprived smokers--a qualitative study. BMC Health Serv Res 2006;6:147.

31. Zakrisson AB, Theander K, Anderzén-Carlsson A. The experience of a multidisciplinary programme of pulmonary rehabilitation in primary health care from the next of kin's perspective: a qualitative study. Prim Care Respir J 2013;22:459-65. 
32. National Board of Health and Welfare. Nationella riktlinjer vid vård av astma och KOL. 2015 http://www.socialstyrelsen.se/Lists/ Artikelkatalog/Attachments/19949/2015-11-3.pdf (cited Jan 24 2018)

33. Ingemansson M. Evidence-based practice for children with asthma in primary care - quality of management and effects of learning Stockholm. Stockholm, Sweden: Karolinska Institutet, 2016.

34. Adams SG, Pitts J, Wynne J, et al. Effect of a primary care continuing education program on clinical practice of chronic obstructive pulmonary disease: translating theory into practice. Mayo Clin Proc 2012;87:862-70.

35. Berggren E, Ödlund Olin A, Orrevall Y, et al. Early palliative home care: Evaluation of an interprofessional educational intervention for district nurses and general practitioners about nutritional care. SAGE Open Med 2017:5:205031211772646.

36. Engström K, Magnusson C, Galanti MR. Socio-demographic, lifestyle and health characteristics among snus users and dual tobacco users in Stockholm County, Sweden. BMC Public Health 2010;10:619.

37. Nacul LC, Soljak M, Meade T. Model for estimating the population prevalence of chronic obstructive pulmonary disease: cross sectional data from the Health Survey for England. Popul Health Metr 2007;5:8.

38. Wass V, Van der Vleuten C, Shatzer J, et al. Assessment of clinical competence. Lancet 2001;357:945-9.

39. Baron RJ. What's keeping us so busy in primary care? A snapshot from one practice. N Engl J Med 2010;362:1632-6. 